\title{
An ultrasound-guided ABCDE approach with a sniff test to evaluate diaphragmatic function without acoustic windows
}

\author{
Latha Y. S. Naik, MBBS, MD - Rakesh V. Sondekoppam, MBBS, MD • \\ J. Jenkin Tsui, BSc $\cdot$ Ban C. H. Tsui, MD
}

Received: 30 March 2016/Revised: 31 May 2016/ Accepted: 10 June 2016/Published online: 20 June 2016

(c) Canadian Anesthesiologists' Society 2016

\section{To the Editor,}

Interscalene block is known to result in phrenic nerve paralysis (PNP) and diaphragmatic dysfunction. The reported incidence of PNP is variable depending on the site of performance and the volume of local anesthetic used. ${ }^{1}$ Blocking the normal hemidiaphragm can lead to significant respiratory morbidity in certain patients, particularly those with preexisting respiratory disease. A simple, easily repeatable bedside test is therefore desirable for assessing diaphragmatic function perioperatively, especially in the context of interscalene block.

Various ultrasound-based techniques described previously to visualize the diaphragm suffer from poor reproducibility, depend on operator experience, or involve measuring the amplitude of motion or change in diaphragm thickness, with subsequent calculations. ${ }^{2}$ The ease of imaging on the left side is challenging because of the limited acoustic window due to the presence of the spleen, making ultrasound techniques less reproducible over the course of the block. $^{2}$ In an attempt to overcome these drawbacks, we developed an ultrasound-guided sniff test to evaluate qualitatively the change in diaphragmatic thickness and the direction of motion in the context of interscalene block.

As illustrated in the Figure, this novel technique utilizes the previously described systematic ABCD approach for evaluating the diaphragm (at the anterior Axillary line, watch $B$ reathing, move the probe $\boldsymbol{C}$ audad, and perform Diaphragmatic Evaluation). ${ }^{3}$ After locating the muscular

L. Y. S. Naik, MBBS, MD - R. V. Sondekoppam, MBBS, MD ·

J. Jenkin Tsui, BSc · B. C. H. Tsui, MD $(\square)$

Department of Anesthesiology and Pain Medicine, University of

Alberta, Edmonton, AB, Canada

e-mail: btsui@ualberta.ca part of the diaphragm, the sniff test is applied, and the change in thickness of the diaphragm noted via both B-mode and M-mode ultrasonography. During the sniff test, we often note that there is a directional motion of the diaphragm on M-mode ultrasonography. When the diaphragm is functioning normally, there is a brief descent of the diaphragm during the sniff, as reflected by a downward spike in $\mathrm{M}$ mode (with the probe directed cephalad). An upward spike in $M$ mode indicates a paradoxical motion that is seen in a paralysed diaphragm (Figure).

It is well known that the change in thickness of the muscular part of the diaphragm is an indicator of diaphragmatic function. By applying a rapid sniff such as that used in our technique, this change is accentuated and can serve as an objective sign of diaphragmatic contraction and a functioning phrenic nerve. The physiological phenomenon underlying paradoxical upward motion can best be explained by the effect of trans-diaphragmatic pressure changes on the paralyzed diaphragm. Normally during inspiration, the diaphragm muscle thickens and moves downward, creating positive intra-abdominal pressure and negative intrathoracic pressure. ${ }^{4}$ This sequence can be appreciated as active thickening and a downward deflection upon inspiration (away from the probe). When one hemidiaphragm is paralyzed (such as during phrenic nerve blockade from an interscalene block), the opposite side tries to compensate to maintain nearnormal trans-diaphragmatic pressure via increased neural drive. It results in upward displacement of the paralyzed side of the diaphragm. These changes are accentuated upon sniffing and are appreciated easily when incorporated as a part of the assessment technique.

Previous investigators have noted the utility of an ultrasound-guided sniff test by looking at the central 

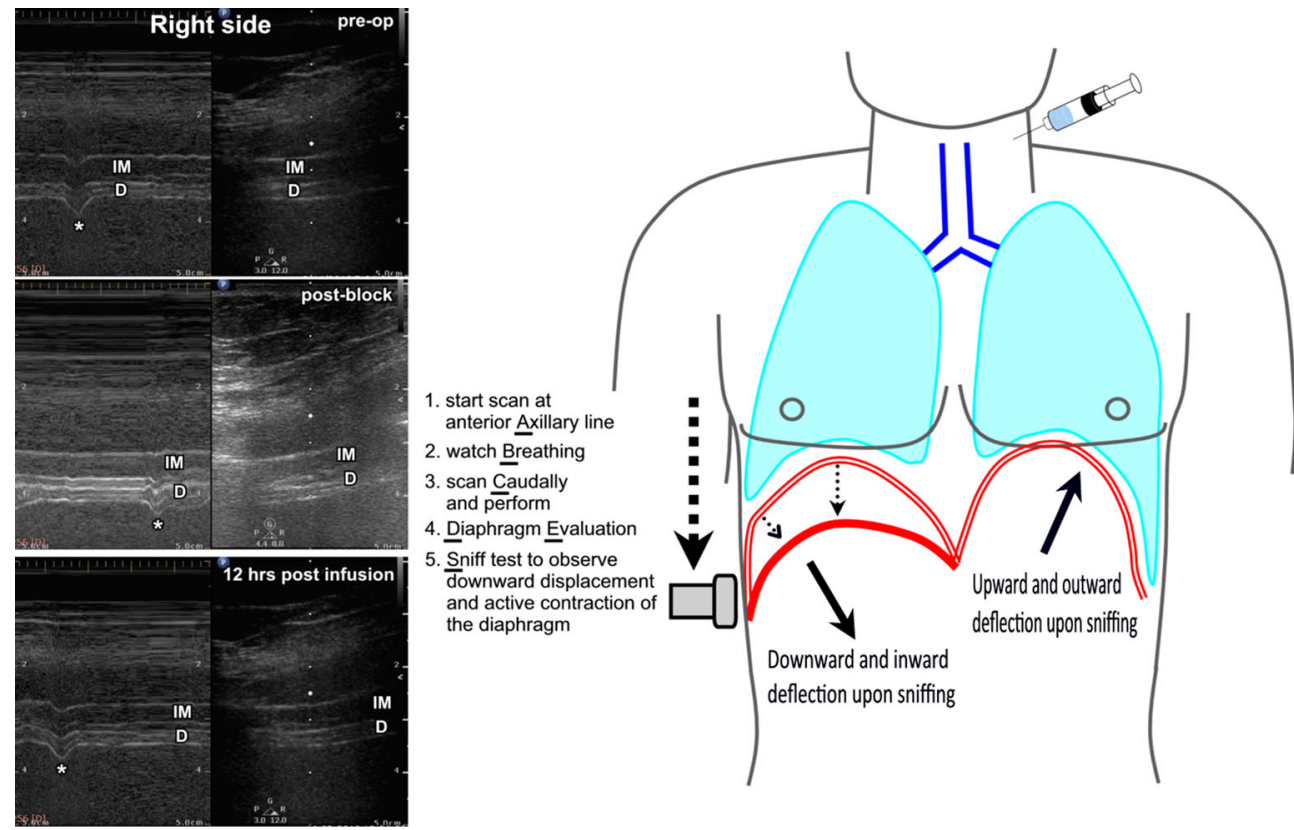

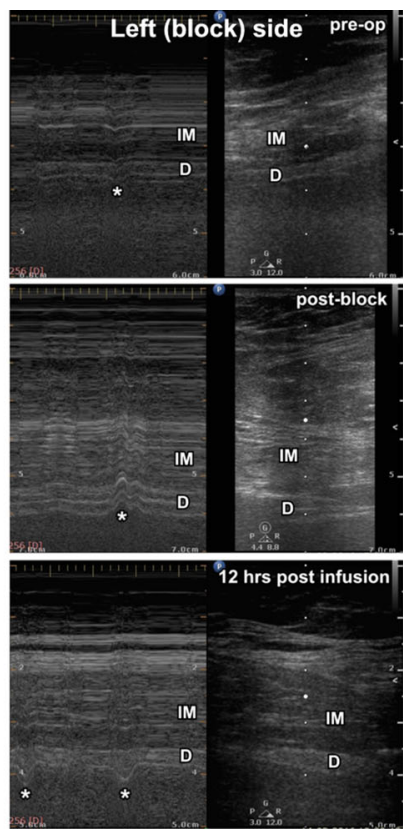

Figure Evaluation of diaphragmatic function in a 62-yr-old, opioiddependent woman $\left(152 \mathrm{~cm}, 130 \mathrm{~kg}\right.$, body mass index $\left.56 \mathrm{~kg} \cdot \mathrm{m}^{-2}\right)$ (written consent obtained) who underwent revision arthroplasty of the left shoulder with continuous interscalene block. M-mode ultrasonographic images of the patient's right (unblocked) and left (blocked) sides upon sniffing preoperatively (top), 30 min after block initiation (middle), and $12 \mathrm{hr}$ after discontinuing the local anesthetic infusion (post-infusion) (bottom). Only in the immediate post-block

tendon of the diaphragm through the splenic or hepatic window. Our technique allows visualisation of the muscular portion of the diaphragm that is superficial and independent of hepatic and splenic windows, thereby providing the advantage of repeatability. It also eliminates the impact of the body habitus and the side-toside variations during scanning. As our technique is an objective measure of function and does not involve measurements and calculations, anesthesiologists may find it easier to adopt in everyday clinical practice.

We believe that the ABCDE method using ultrasonography, combined with the sniff test, could be a useful tool for detecting diaphragmatic dysfunction. Further, well-designed studies are necessary to determine the merit of this technique.

Disclosure Dr. Tsui is supported by a Clinical Scholar Award from the Alberta Heritage Foundation for Medical Research.

Conflicts of interest None declared. scan, an upward deflection of the diaphragm is seen on the left side upon sniffing. Normal downward deflection is observed on the right side. $\mathrm{D}=$ diaphragm; $\mathrm{IM}=$ intercostal muscles. The asterisk indicates the time at which the sniff maneuver was performed. The schematic diagram in the centre describes a positive ABCDEs test (at the anterior Axillary line, watch Breathing, move the probe Caudally, Diaphragm Elevation followed by the sniff test) for evaluating diaphragmatic function.

Editorial responsibility This submission was handled by Dr. Gregory L. Bryson, Deputy Editor-in-Chief, Canadian Journal of Anesthesia.

\section{References}

1. Verelst $P$, van Zundert A. Respiratory impact of analgesic strategies for shoulder surgery. Reg Anesth Pain Med 2013; 38: 50-3.

2. Sarwal A, Walker FO, Cartwright MS. Neuromuscular ultrasound for evaluation of the diaphragm. Muscle Nerve 2013; 47: 319-29.

3. Tsui JJ, Tsui BC. A novel systematic ABC approach to Diaphragmatic Evaluation (ABCDE). Can J Anesth 2016; 63: 636-7.

4. Caruso P, Albuquerque AL, Santana PV, et al. Diagnostic methods to assess inspiratory and expiratory muscle strength. J Bras Pneumol 2015; 41: 110-23. 